\title{
The Heavens in October
}

\section{Recapture of the Tiny Planet 1911 MT.}

\section{By Henry Norris Russell, Ph.D.}

F ASILY first in interest among the astronomical $\mathrm{L}_{\text {announcements of the past summer is the recap- }}$ ture-if we may so speak-of the very remarkable asteroid known as $1911 \mathrm{MT}$.

The history of this little body has afforded qutte हn astronomical romance, which nearly turned out, as has been well said, to be an astronomical tragedy.

On October 3d, 1911, Prof. Palisa of Vienna, a distinguished observer of the minor planets, while observing another object, noticed that one of a pair of faint stars in the field of view of his telescope had changed its position relative to the other. A few minutes measures showed which one was moving, and made it clear that an object of unusual interest had been discovered; for the new asteroid was moving rapidly southward and eastward.

The little planet was almost in opposition to the Sun; and when in this relative position, practically all the known planets and asteroids seem to move westward. They are all really moving eastward, it is true, around the Sun; but the eastward motion of all previously known planets (except Eros near perihelion) is slower than that of the Earth,' so that we leave them behind, and they seem to move toward the west.

The news of the discovery was spread abroad by telegraph, and on the following night another observation was secured by Palisa, and two by Pechule at Copenhagen, but then bad weather set in; the Moon came along and made the sky too bright to see faint stars; and, after she had gone, the new planet was lost, and could not be recovered, though photographs of the region in which it might be expected to be, were taken with powerful instruments.

So the situation remained for several months. The existing observations of the asteroid were confined to two days, and did not afford enough material for the computation of a reliable orbit by the ordinary methods. However, an attack on the problem was made by Mr. Haynes of the University of California, using certain very powerful methods of calculation developed by Prof. Leuschner of that university, and he succeeded in getting an approximate orbit, which was published last April. From this an approximate ephemeris of the planet's tract was cal culated, and, when this was available, images of the planet were found on three plates taken at Greenwich on october 11th, and on one obtained at Heidelberg on October 17th. These images were faint, and had previously been overlooked, but wer found when it was know: approximately where to search for them.

With the aid of these obseryations, Mr. Haynes has computed a second and much more accurate orbit and an improxed seare ephemeris. Thanks to this, the planet has been identified on a plate taken at Heidelberg in the middle of September, and one dle of September, and one exposed at Johannesburs on October 18th. With this interval of a month between our extreme observations, a good orbit can be obtained; and it is possible that still more observations may turn up,
for the planet was well for the planet was well sky, and fully as bright as at discovery, all through August and September, and more images may be found on plates exposed found on plates exposed for other purposes to the
proper region of the sky proper region of the sky-
The orbit of this remarkable member of our solar system is shown in the accompanying illustration. It is remarkable for its great eccentricity, which exceeds that of any previously known planet. When nearest to the Sun, it is but $110,000,000$ miles from him, and about $19,000,000$ from the Earth's orbit, while its greatest distance from the Sim is $366,000,000$ miles, and its distance from the Earth may reach $459,000,000$

The orbit is inclined nearly 11 degrees to the plan



Orbits of the Earth and Planet 1911 MT.

of the ecliptic, crossing it at the points marked $A$ and $\boldsymbol{B}$ in the figure, so that when nearest the Sun it north of the Earth's orbital plane, and a little farther from our track than the diagram would indicate.

At the time of discovery it had just passed as near the Earth as it could possibly come-as the figure shows, by giving the positions of the two bodies on October 3d, 1911 (the discovery date) and on August 29th, when the planet was nearest the Sun. Th planet's motion in this part of its orbit is actually faster than the Earth's motion, which accounts for it apparent eastward motion in the sky. As the two swung farther around their paths they rapidly separated, which caused a steady decline in the apparent

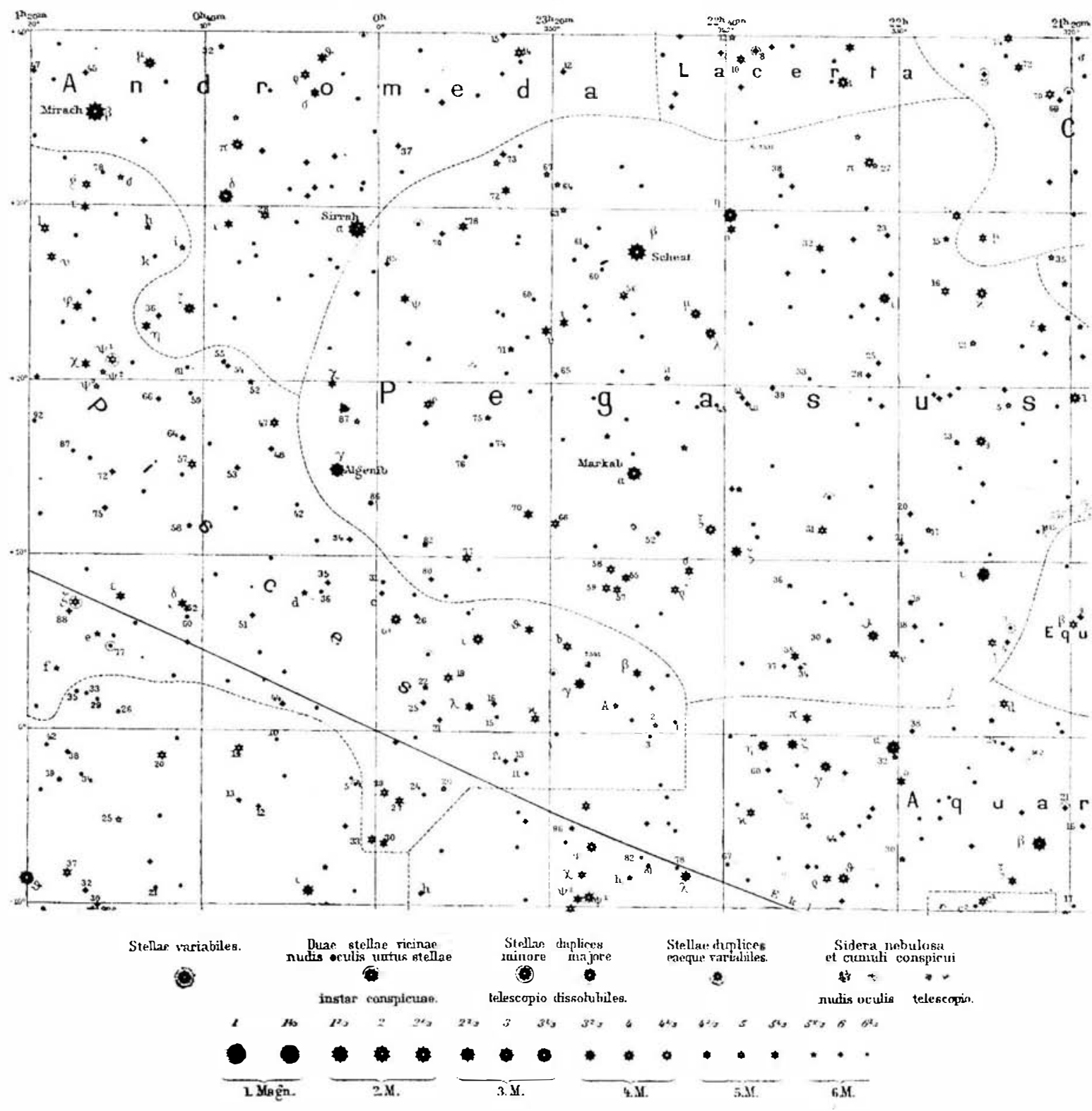

THE HEAVENS IN THE REGION OF PEGASUS AND PISCES brightness of the planet, which explains why it was so nearly lost:

This little planet must be one of the very smallest bodies so far known to astronomical observation. When discovered, dita distance of only $23,000,000$ miles from us it appeared to be of the twelfth magnitude, in spite of the very favorable conditions. If in opposition, at its average distance from the Sun, it would be more than one hundred times fainter, and of magnitude 17.5, hardly visible with the great Yerkes telescope, and just accessible photographically with the most powerful instruments, if its apparent motion were not so rapid. When remotest from the Sun, its magnitude, even if the Earth was most favorably placed, would be 19.8so faint that, even if fixed in the heavens, it could be photographed only with the great Mount Wilson re flector; and, considering its motion, would be beyond even its powers.

It can probably be observed only when in the nearest third of its orbit to the Sun, and then only when the Earth happens to be on the same side of the Sun as the planet. According to Mr. Haynes' calculations, it should return to its perihelion in October, 1915; but observations covering only two weeks give but a slender basis for prediction of a planet's place four years ahead, and it may be necessary to keep a sharp lookout (photographically) on the planet's track for months around this date.

The actual diameter of the new .planet can only be estimated by comparing the amount of light which it reflects with that which we would receive from Mars or Mercury at the same distance; and in this way it may be estimated that it is only three or four miles in diameter, a mere speck compared with the extent of interplanetary space.

The curious name of this little body deserves explatuation. When the discoveries of asteroids became numerous, fifteen years or so ago, they were provisionally denoted by letters; $A, B, C$, etc., the assignment of names and of permanent numbers being reserved until reliable orbits could be calculated. The alphabet was soon exhausted; then pairs of letters were used- $A A, A B$
to $A Z, B A, B B$, etc. Two or three years ago this arrangement ran out; and then it was decided to begin again with $A A$, but to prefix the date of discovery. Hence, the present planet is called $1911 M T$ to distinguish it from one with similar letters in the earlier sequence, disco $\%$ ered some years ago. It will probably not be long before it receives a permanent number and a name.

The principal astronom ical event of the coming month will be the total eclipse of the Sun on October 10th, which is visible in a small part of the southeastern United States as a partial eclipse just after sunrise. The track of total obscuration begins in the Pacific, off the coast of Nicaragua, eross es South America from one point in Ecuador a little north of Quito to one in Brazil a little south and west of Rio de Janeiro. after which the whole course is lost in the South Atlantic. The greatest observable duration of totality is about 1 minute 51) seconds, on the Brazilian coast. It is to be hoped that weather conditions may favor the observers of this eclipse better than they did last year in the T ong a Islands, ' wh e clouds almost completely (Concluded on page 290.) 


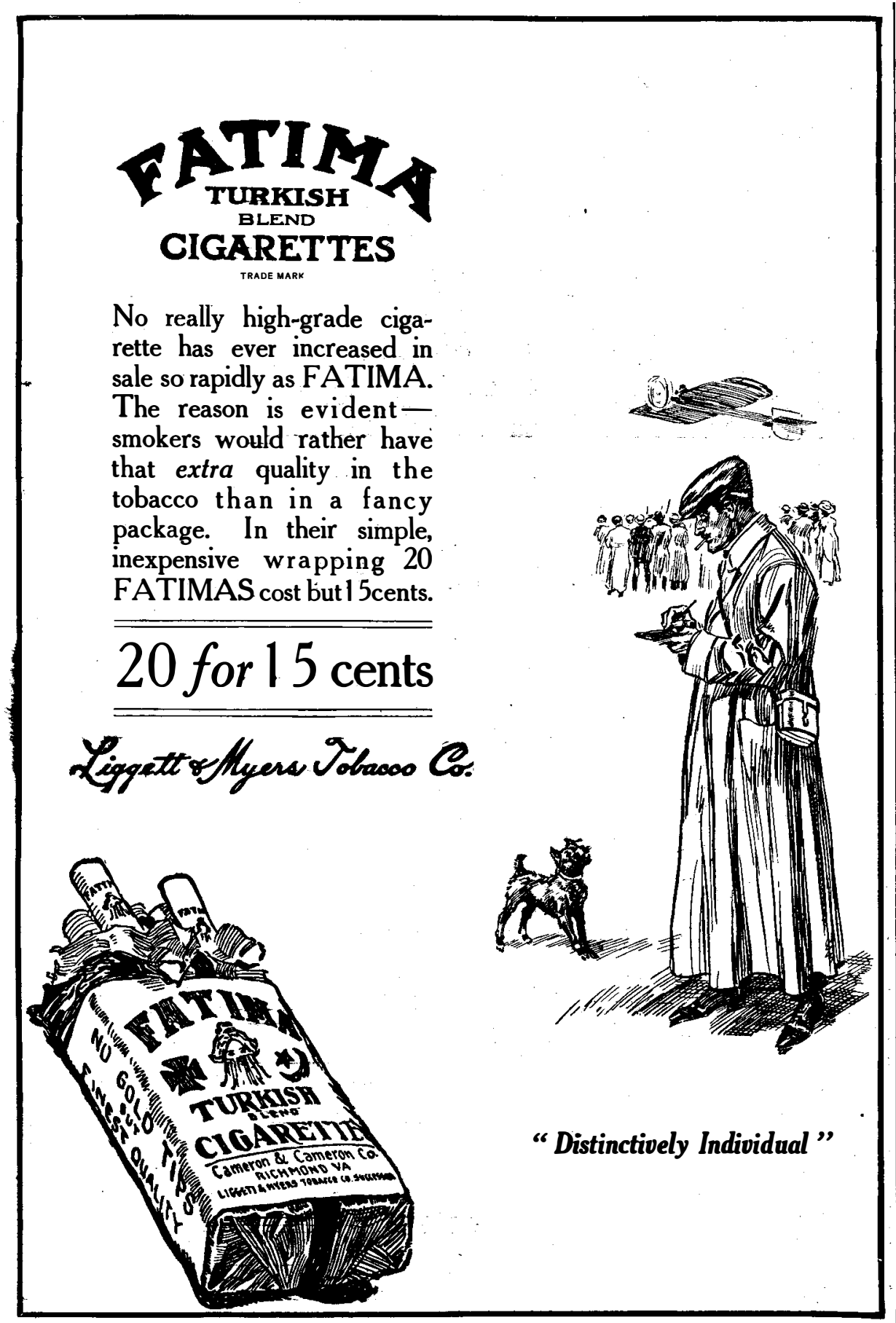

\section{The Panama Canal}

\section{Issue of November 9, 1912}

The time is drawing nigh when the Panama Canal will cease to be a vision and will become a reality. No engineering work in the entire history of the world is of greater magnitude. For decades statesmen and engineers have urged the digging of a waterway which would unite the Pacific with the Atlantic.

The Panama Canal is a monument to American engineering genius. To describe it adequately the Scientific American will devote to it the issue of November 9th-the November magazine number. How the great locks were constructed in which ocean-going steamers will swim, how a mountain was cut in two to make way for the Canal, how great steam shovels that scoop up five tons of dirt at a time dug out the bed of the waterway, and how landslides-avalanches, containing millions of cubic yards-were coped with, all this will be told. Our editor, Mr. J. Bernard Walker, made a special trip to Panama in order to gather much of the material which will appear in this number. He spent several weeks at the Isthmus, studied every square inch of the ground, spoke with the men who are doing the great work-Col. Goethals, Col. Gorgas, and the rest-and brought with him facts which have not yet found their way into print, and which will first appear in the Scientific American. consumed. You find that by making the cost to the consumer bear some relation to the cost of rendering the service, you stimulate the use of current during the day when your plant would ordinarily be loaded but very little, and thus by encouraging the use of current for many hours each day you have put your plant on a self-supporting basis, and have satisfied the consumers by giving them a lower average rate for current because they use it for other than lighting purposes.

You no longer fear that you will be seriously embarrassed by the adoption of high efficiency lamps on the part of you customers; first, because the reduction in units would reduce the number charged at 5 cents, rather than the number charged at 12 cents; and second, because with a well developed heating and cooking load you are not so entirely.dependent upon lighting for your revenue. You therefore, feel in a position to urge you customers to use the high efficiency lamps. You know that they will reduce the customers' bills somewhat, but not enough to cause you to operate at a loss, and on the other hand your customers will greatly appreciate the increased light which these lamps will give at the same time that they reduce the bills. Electric light is thus made cheaper, and is able to compet with gas, not only as far as convenience and safety are concerned, but in cost as well, and the customer finds it possible to use many electrical devices which he would have otherwise found prohibitive from the cost standpoint:

\section{The Heavens in October} (Concluded from page 989)

obscured the Sun, though other parts of the sky were clear.

\section{The Heavens.}

We give again this month a detailed map of a portion of the sky which is now well observable in the evening. The principal configuration shown in it is the great square of Pegasus, near the middle Apart from this, it is one of the poore regions of the sky, being remote from th Milky Way, and rather sparsely sow with stars. Among double stars in this region visible in a small telescope we mav note Epsilon Pegasi (not far from the western edge of the map, just south the parallel of 10 degrees declination) which has a companion of the ninth magnitude, more than two minutes of arc away-too wide to be really called a double star, but a pretty object; $\mathrm{Mu}$ Cygni (about 20 degrees north of the last) a binary pair, now separated by about seconds and closing up, and Zeta Aquarii; another fine slow binary pair separated by 3 seconds, which may be found almost on the celestial equator in 22 hours 22 minutes Right Ascension.

of the constellations in other parts of the sky, Cetus and the Southern Fish are conspicuous, low in the south; Aquila Cygnus and Lyra in the west; Ursa Minor and Draco in the north, with Ursa Majo on the horizon, Perseus and Auriga in the northeast, and Aries and Taurus in the east, while Gemini and Orion are rising.

The Planets.

Mercury is theoretically a morning sta until the 4th, and an evening star afte ward; but he is too near the Sun to be observable.

Venus is evening star in Libra and Scorpio, setting about 6:30 P. M. in the middle of the month, and visible in the twilight.

Mars is approaching conjunction wit the Sun, and though theoretically an eve ing star, cannot be observed.

Jupiter is evening star in Ophiachus setting about 7:50 P. M. on the 15th. Saturn is in Taurus, approaching opposition, rises at $7 \cdot 20 \mathrm{P}$. M. on the $15 \mathrm{t}$ and is well observable in the latter part of the evening.

Uranus is in Capricornus. He is in quadrature with the Sun on the 230, crossing the meridian at $6 \mathrm{P}$. M.

Neptune is in Gemini, and is also in quadrature, on the opposite side of the Sun, on the 19th.

The Moon is in her last quarter at
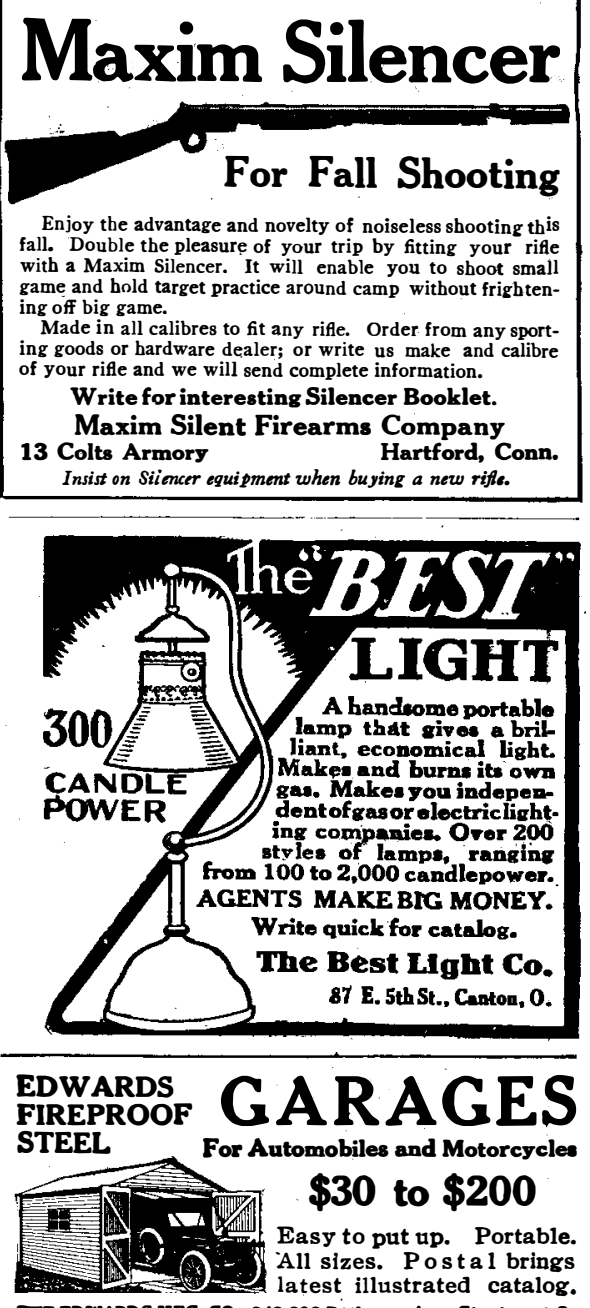

THE EDWARDS MFG. C0., 242-292 Bidte

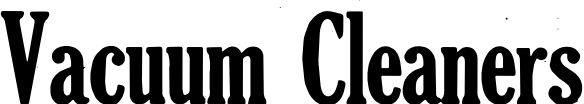

Electric Stationary and Portable, Country Homes special for use with Gasoline Engine

VICTOR CLEANER COMPANY, Manufactarers, YORK, PA. WELL DRILLING

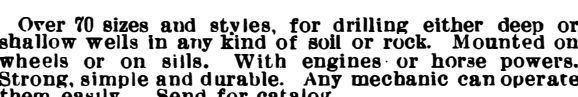
WILLIAMS BROS., Ithaca, N.Y.
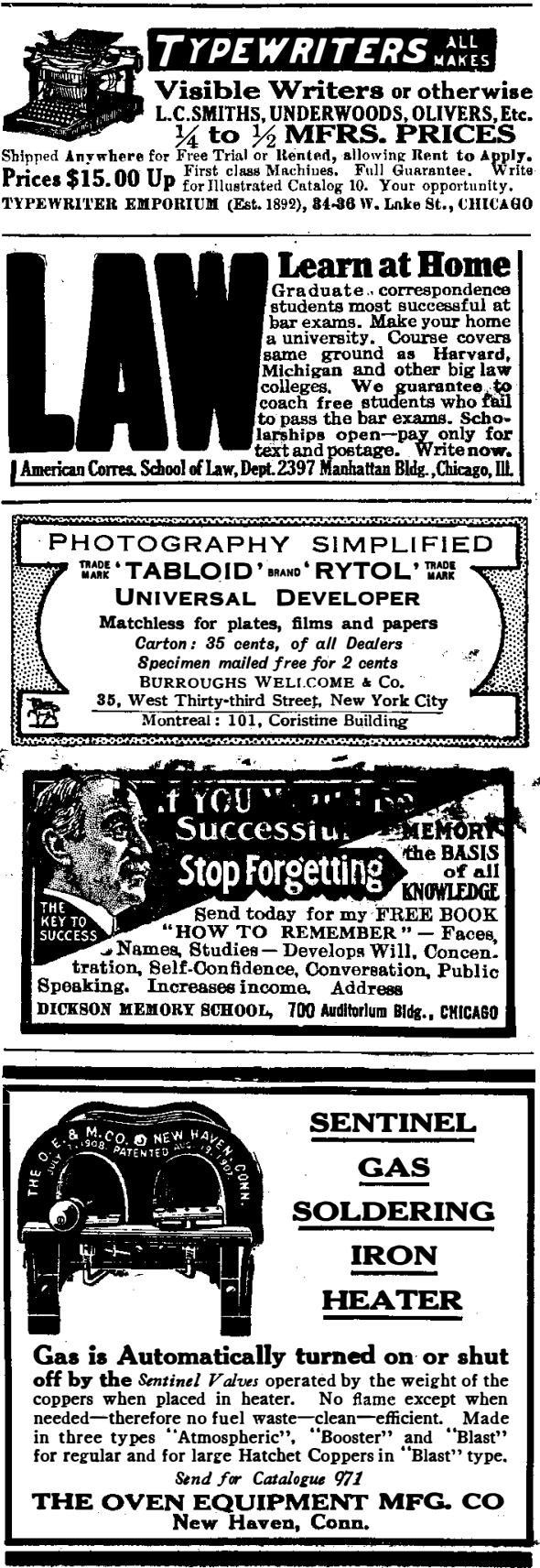
